\title{
Chiari Type 1 malformation presents with extensive periodic breathing pattern in a 3-year-old child
}

\author{
Christine Campbell ${ }^{1}$ and Mariam Ischander ${ }^{1}$ \\ ${ }^{1}$ Western Michigan University
}

June 23, 2020

\begin{abstract}
Periodic breathing is indicative of immaturity of respiratory control and is well recognized within the normal limits in both full term and premature infants but unusual beyond infancy and at high altitude. We would like to focus on the importance of recognizing and scoring the pattern of periodic breathing in older children both clinically and in the sleep laboratory as an abnormal breathing pattern that should draw the attention of the treating physician into the possibility of hindbrain malformation or other significant pathology. Despite that sleep disordered breathing including central and obstructive apneas with or without hypoventilation are well reported as a manifestation of Chiari type I malformation, scoring periodic breathing pattern beyond infancy has not been an area of focus.
\end{abstract}

A 3-year-old female child presents to sleep clinic with a concern of new onset of "deep breaths and gasps" during sleep followed by normal breathing followed by stopping breathing and repeating. Episodes happen about every 3 minutes when sleeping on her back, however these breathing changes do not happen when she sleeps on her right side. Both parents deny any night terrors, or nightmares. Both parents have noticed these apneic episodes during night sleep and naps for the last four months.

Patient has a history of late prematurity born at 36 weeks of gestation by normal vaginal delivery with history of in utero tobacco exposure. There have been no concerns with eating habits, GERD, delays in growth or development. There is no change in voice, difficulty swallowing, notable weakness of arms or abnormal arm movement. Parents have not noticed any truncal ataxia and no reported headaches.

On examination, patient is on the $20^{\text {th }}$ percentile for weight, $22^{\text {nd }}$ percentile for length and $26^{\text {th }}$ percentile for BMI. Physical examination was positive for mildly enlarged nasal turbinates and tonsils 1-2+ with palpable thyroid. Ultrasound of thyroid showed likely fatty tissues with no compression of surrounding structures. Normal muscle strength and normal gait noted.

Initial polysomnogram study was done on room air (see figure 1) and showed severe mainly central sleep disordered breathing with a total apnea hypopnea index (AHI) of 27.4 events per hour (205 central apneas, 6 obstructive apneas, 3 mixed apneas and 7 hypopnea events), extensive pattern of periodic breathing noted during the study and occupied about $7.5 \%$ of total sleep time. Most of the periodic breathing occurred in NREM, specifically in stage 2 however periodic breathing still occurred but to less extent during REM. No snoring was noted. The lowest oxygen saturation was $87 \%$ without any sleep related hypoxemia and no sleep related hypoventilation (peak PCO2 of $45.8 \mathrm{mmHg}$ ). The sleep disordered breathing was slightly worse during REM sleep with REM related apnea-hypopnea index of 30.8 events per hour; the supine apnea-hypopnea index was 24.6 events per hour. The longest apnea event was obstructive in nature and lasted 21.3 seconds. The central apnea events varied from 9 to 17.2 seconds. Periodic limb movements (PLM) noted with PLM index of $4.3 /$ hour. The sleep efficiency was normal at $94.3 \%$ and the sleep architecture was also normal for 
age. Gene sequence analysis of PHOX2B for congenital central hypoventilation syndrome was done due to the severe central apnea and the significant periodic breathing pattern and was negative.

Repeat Polysomnogram was performed on 1 LPM oxygen by simple nasal cannula 10 days after initial sleep study with improvement in central sleep disordered breathing noted on oxygen therapy. During the second polysomnogram, total AHI was 0.5 event per hour, the peak TCO2 was $49 \mathrm{mmHg}$ and there was no sleep related hypoventilation. The lowest oxygen saturation was $93 \%$ without sleep related hypoxemia, no snoring noted, and the sleep efficiency and architecture were both normal for age. Based on this improvement with supplemental oxygen, patient was placed on oxygen during night sleep and naps with a pulse oximeter monitoring at home pending definitive intervention.

Due to the concern of the extensive pattern of periodic breathing in the setting of severe central sleep disordered breathing, MRI brain with and without contrast (see Figure 2) was performed. MRI -showed cerebellar tonsils extended below foramen magnum by $2 \mathrm{~cm}$ with marked crowding of foramen magnum and severe restriction of CSF flow through foramen magnum. Neurosurgery was consulted and patient underwent Suboccipital Craniectomy, First and Second Cervical Laminectomies and Duraguard Duraplasty for Chiari I Decompression. Patient was observed in the PICU overnight and had appropriate oxygen saturations on room air. The surgery was uncomplicated, and patient had resolution of apneic events during sleep.

Repeat polysomnogram on room air was done about 3 months after surgical intervention and showed significant improvement in the sleep disordered breathing and periodic breathing pattern with a total AHI of 1.8 per hour (total of 15 central apnea events without any obstructive or mixed events), and periodic breathing for $2.5 \%$ of total sleep time. The lowest oxygen saturation was $94 \%$ without sleep related hypoxemia and the peak PCO2 was $48 \mathrm{mmHg}$ without sleep related hypoventilation. Normal sleep efficiency and sleep architecture for age noted. Patient clinically continued to do well post-operatively and growing and developing appropriately for age.

\section{DISCUSSION:}

Chiari Type 1 malformation is known to be associated with obstructive sleep apnea and or central sleep apnea (CSA) with or without hypoventilation. This association has been documented in numerous studies with multiple hypotheses as to why it occurs however periodic breathing has not been an area of focus. Periodic breathing is described as a recurrent central apnea events with intermittent respiratory effort ${ }^{1}$ and it is indicative of instability of respiratory control. Periodic breathing is well described in all premature infants less than $1 \mathrm{~kg}$ birth weight and some normal full-term infants and it gradually fades during the first year of life ${ }^{2}$. The periodic breathing pattern is due to increased gain of feedback from chemoreceptors along with longer latency in feedback causing fluctuation in minute ventilation. ${ }^{3}$ More simply: during the central sleep apnea phase of the periodic breathing the CO2 tension will increase and the oxygen tension will decrease leading to the hyperventilation phase. ${ }^{2,4}$ During the hyperventilation phase the CO2 tension will fall below the apnea threshold and causing respiratory pause to start and so on. The American Academy of sleep medicine recommends scoring respiratory event as periodic breathing if there are $>3$ episodes of central pauses in respiration (absent airflow and inspiratory effort) lasting $>3$ seconds and separated by $<20$ seconds of normal breathing. ${ }^{3}$ Central sleep apnea that occurs within the run of periodic breathing should be scored as individual apnea as well. Oxygen has a role in regulation of breathing with improvement in central apnea and periodic breathing in children placed on supplemental oxygen during sleep. ${ }^{5}$ Our Patient had an extensive pattern of periodic breathing during her initial sleep study which is unusual at age 3 years. Central sleep apnea/ periodic breathing in early infancy is normal finding however beyond infancy these findings would be abnormal, and a thorough work up would be indicated. Although neurological disorders, more specifically Chari Malformations, a known cause of CSA in a non-preterm pediatric patient, other etiologies including genetic, endocrine, gastro reflex disease, epilepsy and anatomical malformations must also be considered.

\section{CONCLUSIONS}

While polysomnography are obtained in children with sleep related breathing disorders, no definitive diag- 
nosis can be made based solely on polysomnography studies as patients found to have CM-1 had multiple variations of polysomnography studies. ${ }^{6}$ Therefore our case report highlights that periodic breathing can be seen in patient with Chiari type 1 malformation.

\section{DISCOLOSURE STATEMENT}

This was not an industry supported study, The Authors have no financial Conflict of interest and no funding received related to this case report.

\section{ACKNOWLEDGMENTS}

The authors acknowledge Samantha Williams for technical assistance with this case report and Ann Harris for her assistance with formatting the manuscript reference.

List of Abbreviation:

Chiari Type 1 malformation (CM-1)

Apnea Hypopnea Index (AHI)

Periodic limb movements (PLM)

Central Sleep Apnea (CSA)

REFERENCES:

1. Urquhart DS, Tan HL. Sleep disordered breathing at the extremes of age: Infancy. Breathe. 2016;12(1):e1e11. doi:10.1183/20734735.001016

2. American Academy of Sleep Medicine. The international classification of sleep disorders. 2005.

3. American Academy of Sleep Medicine. The AASM Manual for the scoring of sleep and associated events. Version 2.5. 2018.

4. Katz ES. Disorders of Central Respiratory Control During Sleep in Children. In: Therapy in Sleep Medicine. Elsevier Inc.; 2012. p. 434-447. doi:10.1016/B978-1-4377-1703-7.10034-9

5. Simakajornboon N, Beckerman RC, Mack C, Sharon D, Gozal D. Effect of supplemental oxygen on sleep architecture and cardiorespiratory events in preterm infants. Pediatrics. 2002;110(5):884-888. doi:10.1542/peds.110.5.884

6. Leu RM. Sleep-related breathing disorders and the chiari 1 malformation. Chest. 2015;148(5):1346-1352. doi:10.1378/chest.14-3090

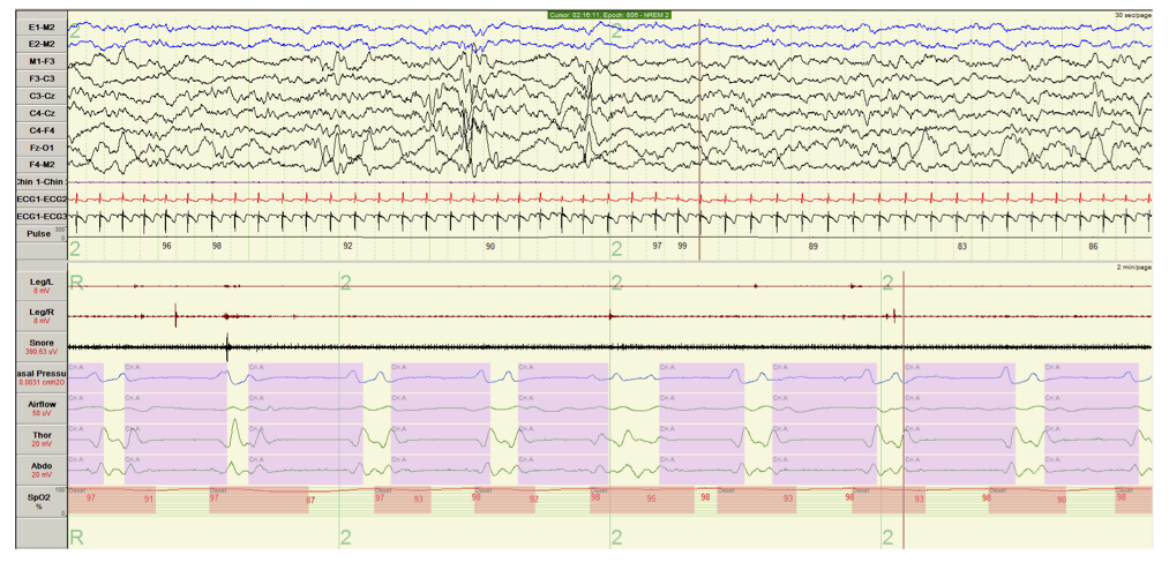

Figure 1 


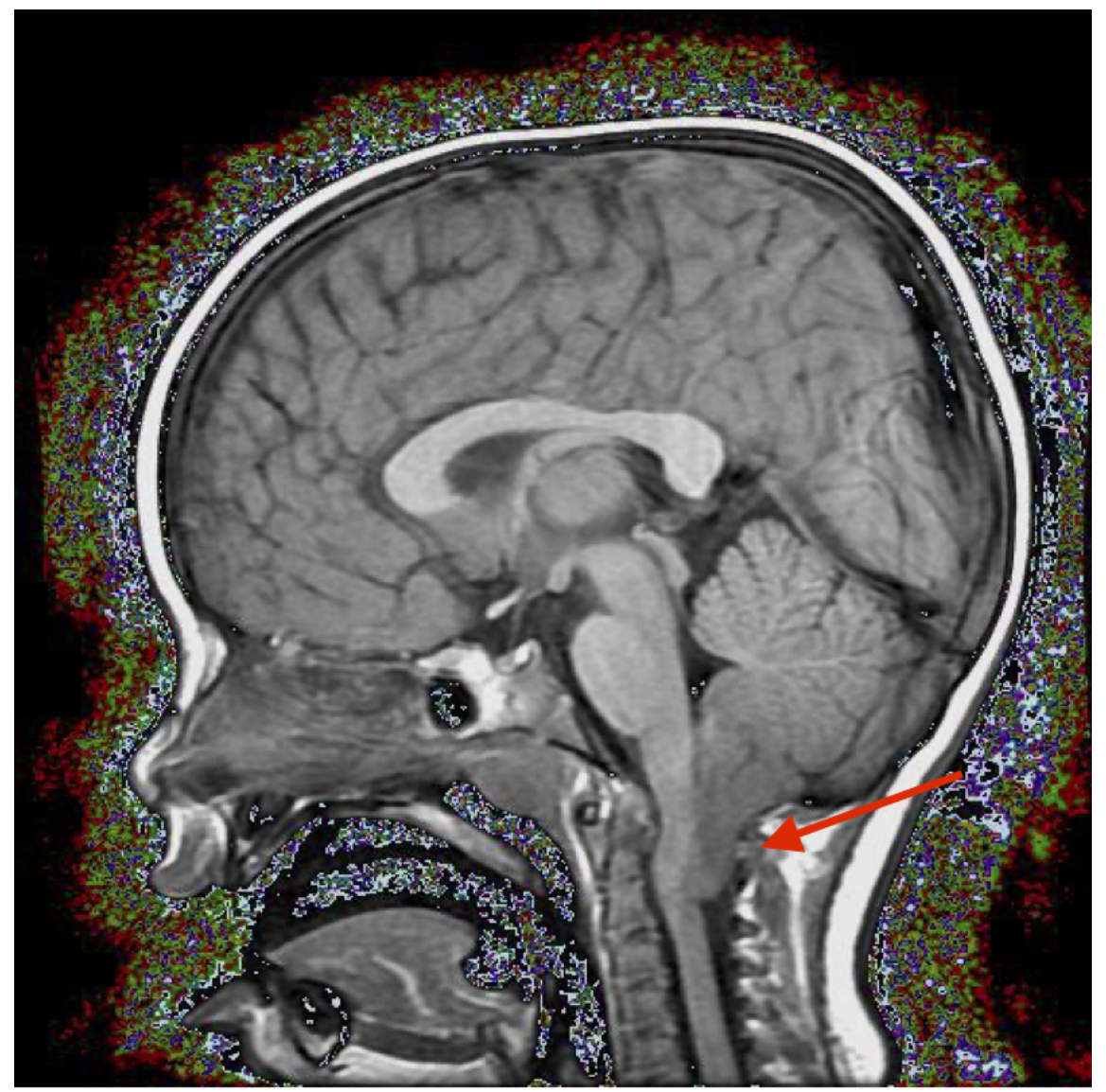

Figure 2: MRI brain with and without contrast. Arrow identifies Chiari Type 1 malformation 